\title{
HEART DISEASE DATA SET CLASSIFICATIONS: COMPARISONS OF CORRELATION CO EFFICIENT BY APPLYING VARIOUS PARAMETERS IN GAUSSIAN PROCESSES
}

\author{
Dr.G.Ayyappan, \\ Associate Professor, Bharath Institute of Higher Education and Research,Chennai \\ ayyappangmca@gmail.com \\ K.SivaKumar, \\ SIPS Technologies, Chennai, India.
}

\begin{abstract}
Data mining, the extraction of hidden predictive information from large databases, is a powerful new technology with great potential used in various commercial applications including retail sales, e-commerce, remote sensing, bioinformatics etc. There are varieties of popular data mining task within the educational data mining e.g. classification, clustering, outlier detection, association rule, prediction etc. This paper focuses Gaussian Proesses of the comparisions various correlation coeffient accuracies by applying the different paprameters pruning methods and analysis in weka tool.
\end{abstract}

Keywords: Classification, Puk Kernel, Poly kernel, Normalized poly kernel, linear kernel,RBF kernel.

\section{INTRODUCTION}

Now a days, large quantities of data is being accumulated. Seeking knowledge from massive data is one of the most desired attributes of Data Mining. Data could be large in two senses: in terms of size \& in terms of dimensionality. Also there is a huge gap from the stored data to the knowledge that could be construed from the data.

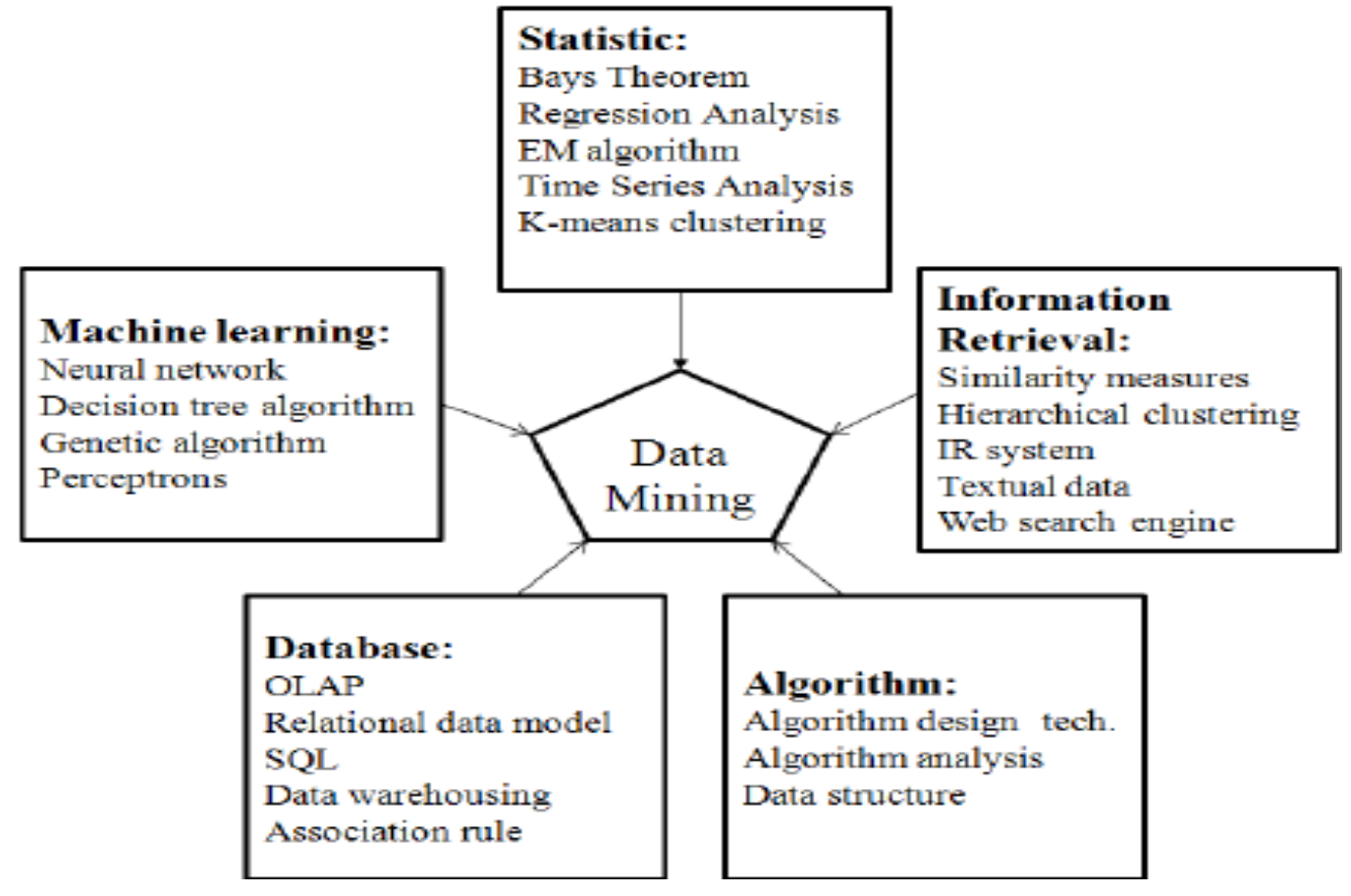

Fig 1:Data Mining 
Manual data analysis has been around for some time now, but it creates a bottleneck for large data analysis. The transition won't occur automatically; in this case, we need the data mining. Data Mining could help in a more in-depth knowledge about the data.

Extreme learning machine (ELM) is a special single-hidden layer feed-forward neural network (SLFN). Due to its lower computational complexity and better generalization performance, ELM has recently attracted a lot of interests in research and industry and is used in a wide range of applications. ELM uses a random method to determine input weights/hidden layer biases and analytically computes the output weights. Therefore, it is extremely fast to train an ELM model. It has also been proved that ELM can guarantee the universal approximate capability of ELM .

In this paper section 1 focuses, introduction about the machine learning, Section 2 presents materials and methods, In section 3 presents results and discussions and finally Section 3 presents conclusion about this research work.

\section{MATERIALS AND METHODS}

In this section presents the materials and methods of this research work. Here it has implemented the weka tool for mining process The WEKA GUI chooser launches the WEKA's graphical environment which has five buttons: Simple CLI, Explorer, Experimenter, Knowledge Flow and Workbench.

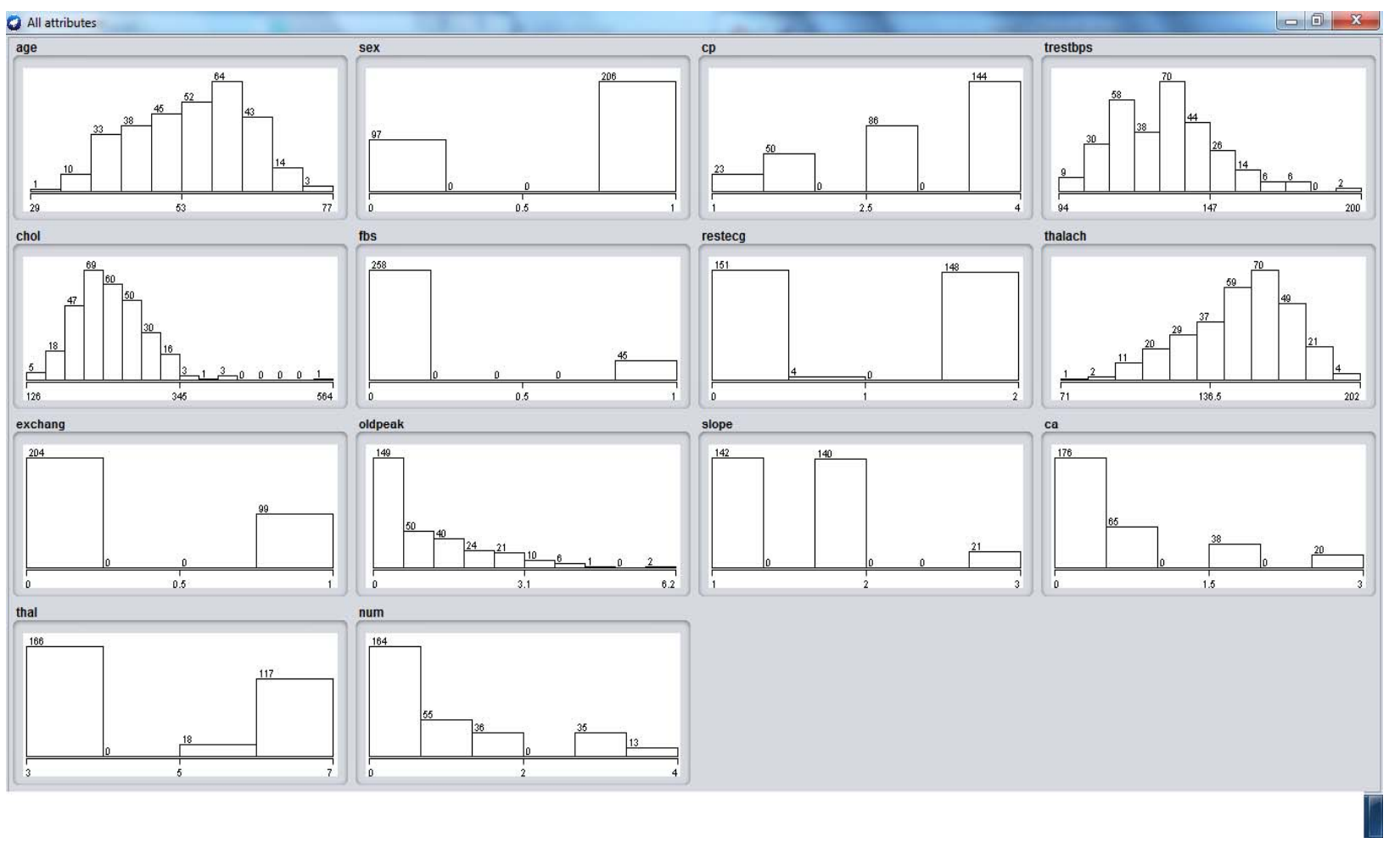

Fig 2:DataVisualization

\section{Dataset Information}

This database contains 76 attributes, but all published experiments refer to using a subset of 14 of them. In particular, the Cleveland database is the only one that has been used by ML researchers to this date. The "goal" field refers to the presence of heart disease in the patient. It is integer valued from 0 (no presence) to 4. Experiments with the Cleveland database have concentrated on simply attempting to distinguish presence (values 1,2,3,4) from absence (value 0 ).

Table 1: Dataset Information

\begin{tabular}{|c|c|c|c|c|}
\hline Name & Data type category & No of attributes & No of Instances & No of Attributes \\
\hline Heart Disease & Multivaiate & $\begin{array}{c}\text { Categorical, Integer, } \\
\text { and Real }\end{array}$ & 303 & 75 \\
\hline
\end{tabular}




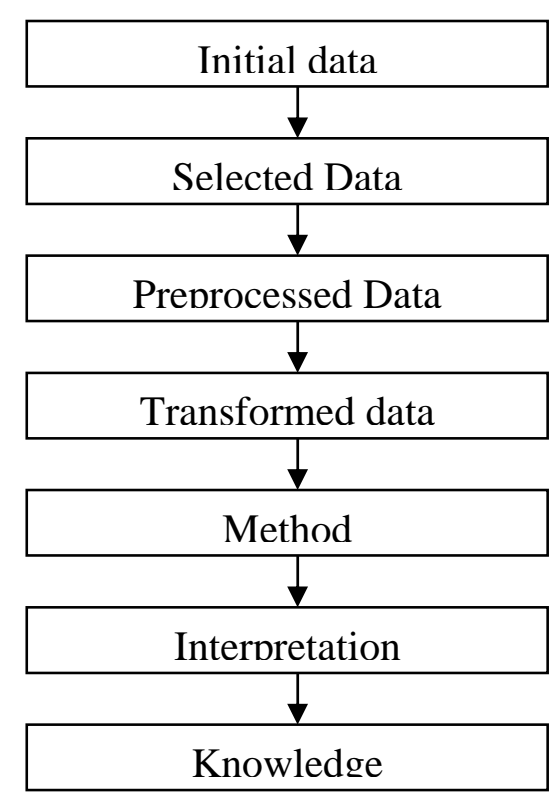

Fig 3:Flow process of Heart Diseas dataset classification

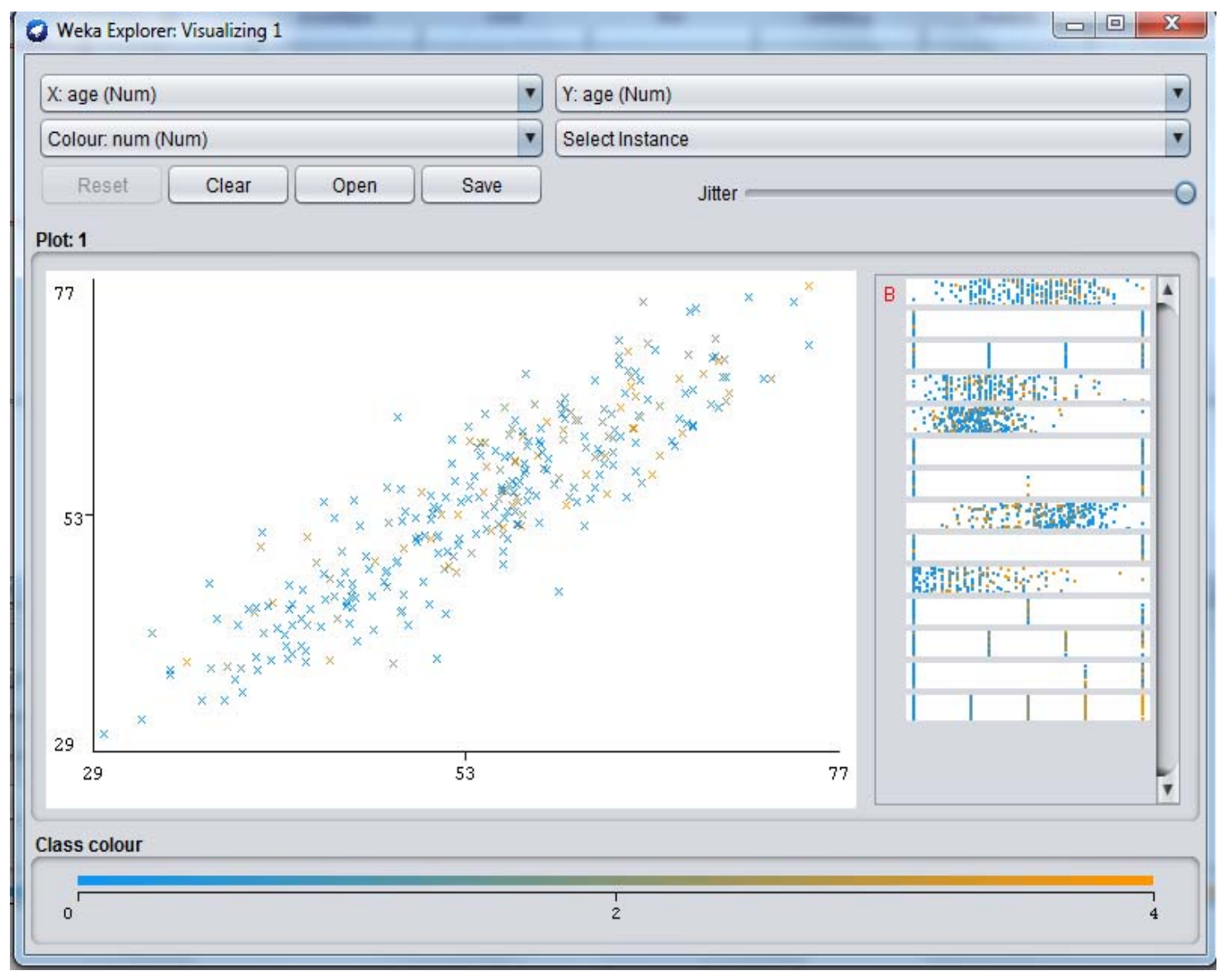

Fig 4:Data visualization based on age of Heart Disease dataset classification 
Table 2: List of Attributes

\begin{tabular}{|l|l|l|}
\hline S.No & Label & Meaning and functions of Lebel \\
\hline 1 & age & age in years \\
\hline 2 & sex & Sex (1=male ; $0=$ female) \\
\hline 3 & trestbps & resting blood pressure(in mm Hg on admission to the hospital) \\
\hline 5 & chol & serum cholestoral in mg/dl \\
\hline 6 & fbs & (fasting blood sugar $>120$ mg/dl) (1=true; $0=$ false) \\
\hline 7 & restecg & resting electrocar diographic results \\
\hline 8 & thalach & maximum heart rate achieved \\
\hline 9 & exang & exercise induced angina(1=yes; $0=$ no) \\
\hline 10 & oldpeak & ST depreve to restse relatission induced by exercise relative to rest \\
\hline 11 & slope & the slope of the peak exercise ST segment \\
\hline 12 & ca & number of major vessels(0-3)colored by flourosopy \\
\hline 13 & thal & 3=normal; 6=fixed defect; $7=$ reversable defect \\
\hline 14 & num & diagnosis of heart disease (angiographic disease status) \\
\hline
\end{tabular}

The above 14 data attributes are processed and it has derived from 76 attributes.

In this research paper implements the various kernel parameters pruning. They are

Linear Kernel: $\mathrm{K}(\mathrm{x}, \mathrm{y})=\langle\mathrm{x}, \mathrm{y}>$

Normalized Poly Kernel: $\mathrm{K}(\mathrm{x}, \mathrm{y})=<\mathrm{x}, \mathrm{y}>\wedge 2.0 /\left(<\mathrm{x}, \mathrm{x}>\wedge 2.0^{*}<\mathrm{y}, \mathrm{y}>\wedge 2.0\right)^{\wedge}(1 / 2)$

RBF Kernel: $K(x, y)=\exp (-0.01 *(x-y) \wedge 2)$

Puk Kernal: The Pearson VII function-based universal kernel.

\section{RESULTS AND DISCUSSIONS}

In this section clearly described the results of applying the various parameters in Gaussian processes and it produces the various co efficient accuracies and also it mentioned the time taken to build the models.

Table 2:Various Correlation coefficient accuracies of Gaussian processes

\begin{tabular}{|c|c|c|}
\hline Kernel & $\begin{array}{c}\text { Accuracy of Correlation } \\
\text { Coefficient }\end{array}$ & $\begin{array}{c}\text { Time taken to build the model } \\
\text { (In Seconds) }\end{array}$ \\
\hline Linear or poly kernel & 0.5145 & 0.56 \\
\hline Normalized poly kernel & 0.5376 & 0.34 \\
\hline Puk kernel & 0.4863 & 0.38 \\
\hline RBF Kernel & 0.4199 & 0.31 \\
\hline
\end{tabular}




\section{Accuracy of Correlation Coefficient}

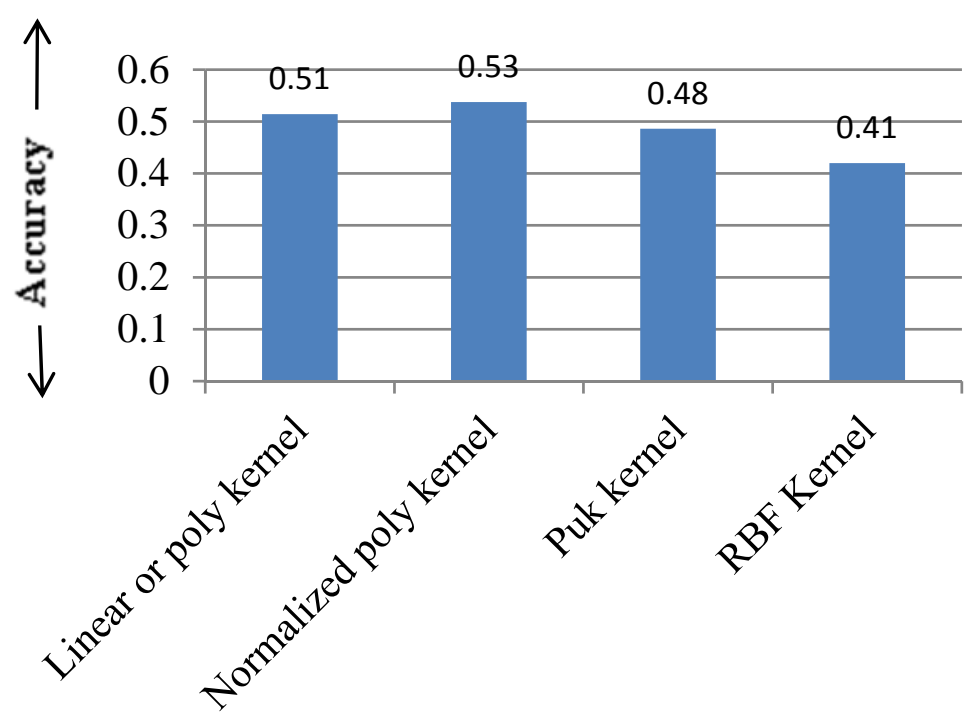

$\longleftarrow$ Various parameters in Gaussian Process-

Fig 4: Various Co Efficient Accuracies

This above diagram represents the various kernel parameters and the models produces the various correlation coefficients in gaussians processes.The model produces the correlation coefficent accuracy level 0.51 while applying linear or poly kernel in this data set. The model produces the correlation coefficent accuracy level 0.53 while applying Normalized poly kernel in this data set. The model produces the correlation coefficent accuracy level 0.48 while applying linear or poly kernel in this data set. The model produces the correlation coefficent accuracy level 0.41 while applying RBF kernel in this data set.

\section{CONCLUSIONS}

The results clearly demonstrate the various parameters pruning for Gaussian processes correlation coefficient accuracies and the model discovered the time has taken to build the models. The Normalized poly kernel has the highest accuracy compare than other accuracy results. So the recommended model is normalized poly kernel model in Gaussian process.

\section{REFERENCES}

[1] Erdogan and Timor (2005) A data mining application in a student database. Journal of Aeronautic and Space Technologies July 2005 Volume 2 Number 2 (53-57)

[2] Romero C. and Ventura S.,"Educational data mining: A Survey from 1995 to 2005".Expert Systems with Applications (33) 135-146. 2007

[3] https://www.researchgate.net/publication/266602921_Data_Mining_in_Educational_System_using_WEKA.

[4] https://archive.ics.uci.edu/ml/datasets/Heart+Disease

[5] W. Huber, On the use of the correlation coefficient $r$ for testing the linearity of calibration functions. Accreditation and Quality Assurance 9 (2004):726-727.

[6] http://www.dtic.mil/dtic/tr/fulltext/u2/1015871.pdf

[7] Wong, K.I., Vong, C.M., Wong, P.K., Luo, J.H.: Sparse Bayesian extreme learning machine and its application to biofuel engine performance prediction. Neurocomputing 149, 397-404 (2015)

[8] Matias, T., Souza, F., Ara'ujo, R., Antunes, C.H.: Learning of A Single-Hidden Layer Feedforward Neural Network Using An Optimized Extreme Learning Machine. Neurocomputing 129, 428-436 (2014)

[9] Huang, G.B., Chen, L., Siew, C.K.: Universal Approximation Using Incremental Constructive Feedforward Networks with Random Hidden Nodes. IEEE Trans. Neural Netw. 17(4), 879-892 (2006)

[10] Huang, G.B., Wang, D.H., Lan, Y.: Extreme Learning Machines: A Survey. Int. J. Mach. Learn. \& Cybern. 2(2), 107-122 (2011)

[11] Huang, G.B., Zhu, Q.Y., Siew, C.K.: Extreme Learning Machine: Theory and Applications. Neurocomputing 70(1), 489-501 (2006). 